\title{
Classification of Self-Organizing Hierarchical Mobile Adhoc Network Routing Protocols - A Summary
}

\author{
Udayachandran Ramasamy, \\ Professor \& Head, \\ Department of Computer Science \& Engineering \\ Sri Ramakrishna Institute of Technology, \\ Coimbatore - 641 010, India, \\ Email : ruday2004@yahoo.co.in
}

\author{
K. Sankaranarayanan \\ Principal, \\ Akshaya College of Engineering and Technology \\ Kinathukadavu, Coimbatore - 642 109, India \\ Email : kkd-sankar@yahoo.com
}

\begin{abstract}
MANET is a special kind of wireless network. It is a collection of mobile nodes without having aid of established infrastructure. Mobile Adhoc network removes the dependence on a fixed network infrastructure by treating every available mobile node as an intermediate switch, thereby extending the range of mobile nodes well beyond that of their base transceivers. Other advantages of Manet include easy installation and upgrade, low cost and maintenance, more flexibility, and the ability to employ new and efficient routing protocols for wireless communication. In this paper we present four routing algorithm, classifications, discuss their advantages and disadvantages.
\end{abstract}

Keywords- MANET, Routing Protocols, Routing Topology , Routing Algorithms and QoS.

\section{INTRODUCTION}

Suppose it is required to easily and effectively connect two office floors using short range wireless communication devices. Every employee has one of these mobile devices, and some fixed devices- computers, printers, and so on-have the same capability.

It is possible to connect these devices to the existing wired infrastructure using access points, but this option offers limited mobility, adds load on the wired networking and relies on existing protocols for wired communication. Another possibility is to build a network of dedicated and mutually connected base stations that enable cellular communication, but this is expensive with respect to time, installation, and maintenance.

The best solution is to create a mobile Adhoc network using surrounding electronic devices as intermediate switches when they are idle and if they are capable of performing this task. For example, the packet from one device can hop to the mobile phone of a person passing through the corridor in front of the office, then from the mobile phone to the shared laser printer in the next office, then to someone's digital wristwatch on the floor below, then from the wristwatch to the coffee machine, and, finally, from the coffee machine to its ultimate destination - say another colleague's device or computer. To date, MANETs have been used primarily for military purposes, while commercial applications are just beginning to emerge. One of the potential practical usage scenarios of MANETs is in a conference room where a group of people that possibly have not met before come together for an Adhoc meeting. They may wish to exchange data securely with their notebook computers or PDAs without any additional infrastructure support [1].

Small scale MANETs are also effective for emergency search and rescue, battlefield surveillance and other communication application in hazardous environments. For example, robots or autonomous sensors deployed in an area inaccessible to humans could use simple MANET routing protocols to transmit data to a control centre. Even if many robots or sensors are disabled or destroyed, the remaining ones would be able to reconfigure themselves and continue transmitting information.

\section{ROUTING IN MANETS}

The major challenges that a routing protocol designed for Adhoc wireless networks faces are mobility of nodes, resource constraints, error-prone channel state, and hidden and exposed terminal problems.

Due to the issues in an Adhoc wireless network environment mentioned above, wired network routing protocols cannot be used in Adhoc wireless networks. Hence Adhoc wireless networks require specialized routing protocols that address the challenges described above. A routing protocol for Adhoc wireless networks should have the following characteristics [2].

- It must be fully distributed, as centralized routing involves high control overhead and hence is not scalable. Distributed routing is more fault-tolerant than centralized routing, which involves the risk of single point of failure.

- It must be adaptive to frequent topology changes caused by the mobility of the nodes.

- Route computation and maintenance must involve a minimum number of nodes. Each node in the network must have quick access to routes, that is, minimum connection set up time is desired.

- It must be localized, as global state maintenance involves a huge state propagation control overhead. 
- It must be loop-free and free from stale routes.

- The number of packet collisions must be kept to a minimum by limiting the number of broadcasts made by each node. The transmissions should be reliable to reduce message loss and to prevent the occurrence of stale routes.

- It must converge to optimal routes once the network topology becomes stable. The convergence must be quick.

- It must optimally use source resources such as bandwidth, computing power, memory power, and battery power.

- Every node in the network should try to store information regarding the stable local topology only. Frequent changes in local topology and changes in the topology of parts of the network with which the node does not have any traffic correspondence, must not in any way affect the node, that is, changes in remote parts of the network must not cause updates in the topology information maintained by the node.

- It should be able to provide a certain level of quality of service (QoS) as demanded by the applications, and should also offer to support for time-sensitive traffic.

\section{Classification of Routing Protocols}

Routing protocols for Adhoc wireless networks can be classified into several types based on different criteria. The routing protocols for Adhoc wireless networks can be broadly classified into four categories based on

- $\quad$ Routing information update mechanism

- Use of temporal information for routing

- Routing topology

- Utilization of specific resource

\section{A. Based on the Routing Information Update Mechanism}

Adhoc wireless network routing protocols can be classified into three major categories based on the routing information update mechanism. They are

\section{1) Proactive or Table-Driven Routing Protocols}

In table-driven routing protocols, every node maintains the network topology information in the form of routing tables by periodically exchanging routing information. Routing information is generally flooded in the whole network. Whenever a node requires a path to a destination, it runs an appropriate path-finding algorithm on the topology information it maintains. The table-driven protocols are

DSDV - Destination- Sequenced Distance-Vector [3]

WRP - Wireless Routing Protocol [4]

CGSR - Clustered Head Gateway Switch Routing [5]
STAR - Source Tree Adaptive Routing [6]

OLSR - Optimized Link State Routing [7]

FSR - Fisheye State Routing [8]

HSR - Hierarchical State Routing [8]

GSR - Global State Routing [9]

\section{2) Reactive or On-demand Routing Protocols}

Protocols that fall under this category do not maintain the network topology information. They obtain the necessary path when it is required, by using a connection establishment process. Hence these protocols do not exchange routing information periodically. Some of the existing routing protocols that belong to this category are given below.

DSR - Dynamic Source Routing [10]

AODV - Adhoc On-Demand Distance Vector Routing [11]

ABR - Associativity Based Routing [12]

SSA - Signal Stability Based Adaptive Routing [13]

FORP - Flow-Oriented Routing Protocol [14]

PLBR - Preferred Link-Based Routing [15]

\section{3) Hybrid Routing Protocols}

Protocols belonging to this category combine the best features of the above two categories. Nodes within a certain distance from the node concerned, or within a particular geographical region, are said to be within the routing zone of the given node. For routing within this zone, a table-driven approach is used. For nodes that are located beyond this zone, an on-demand approach is used. Some of the protocols in this category are

CEDAR - Core Extraction Distributed Adhoc Routing [16]

ZRP - Zone Routing Protocol [17]

ZHLS - Zone-Based Hierarchical Link State Routing [18]

\section{B. Based on the Use of Temporal Information for Routing}

This classification of routing protocols is based on the use of temporal information used for routing. Since Adhoc wireless networks are highly dynamic and path breaks are much more frequent than in wired networks, the use of temporal information regarding the lifetime of the wireless links and the lifetime of the paths selected assumes significance. The protocols that fall under this category can be further classified into two types:

\section{1) Routing Protocols Using Past Temporal Information}

These routing protocols use information about past status of the links or the status of the links at the time of routing to make routing decisions. For example, the routing metric based on the availability of wireless links (which is the current / present information here) along with a shortest path-finding algorithm, provides a path that may be efficient and stable at the time of path-finding. The topological changes may 
immediately break the path, making the path undergo a resource-wise expensive path reconfiguration process. Some of the protocols in this category are given below.

DSDV - Destination- Sequenced Distance-Vector [3]

WRP - Wireless Routing Protocol [4]

STAR - Source Tree Adaptive Routing [6]

DSR - Dynamic Source Routing [10]

AODV - Adhoc On-Demand Distance Vector Routing [11]

FSR - Fisheye State Routing [8]

HSR - Hierarchical State Routing [8]

GSR - Global State Routing [9]

\section{2) Routing Protocol That Use Future Temporal Information}

Protocols belonging to this category use information about the expected future status of the wireless links to make approximate routing divisions. Apart from the life-time of wireless links, the future status information also includes information regarding the lifetime of the node (which is based o the remaining battery charge and discharge rate of the nonreplenish able resources), prediction of location and prediction of link availability. The protocols in this category are

FORP - Flow-Oriented Routing Protocol [14]

RABR - Route-Lifetime Assessment - based Routing [19]

LBR - Link Life-time based Routing Protocol [20]

\section{Based on the Routing Topology}

Routing topology being used in the Internet is hierarchical in order to reduce the state information maintained at the core routers. Adhoc wireless networks, due to their relatively smaller number of nodes, can make use of either a flat topology or a hierarchical topology for routing.

\section{1) Flat Topology Routing Protocols}

Protocols that fall under this category make use of a flat addressing scheme similar to the one used in IEEE 802.3 LANs. It assumes the presence of a globally unique (or atleast unique to the connected part of the network) addressing mechanism for nodes in an Adhoc wireless networks. These are

DSR - Dynamic Source Routing [10]

AODV - Adhoc On-Demand Distance Vector Routing [11]

ABR - Associatively Based Routing [12]

SSA - Signal Stability Based Adaptive Routing [13]

FORP - Flow-Oriented Routing Protocol [14]

\section{PLBR - Preferred Link-Based Routing [15]}

\section{2) Hierarchical Topology Routing Protocols}

Protocols belonging to this category make use of a logical hierarchy in the network an associated addressing scheme. The hierarchy could be based on geographical information or it could be based on hop distance. Some of these protocols are

CGSR - Clustered Head Gateway Switch Routing [5]

FSR - Fisheye State Routing [8]

HSR - Hierarchical State Routing [8]

D. Based on the Utilization of specific Resources

1) Power-aware Routing

This category of routing protocols aims at minimizing the consumption of very important resources in the Adhoc wireless networks: the battery power. The routing decisions are based on minimizing the power consumption either locally or globally in the network.

PAR - Power-Aware Routing Protocol [21]

\section{2) Geographical Information Assisted Routing:}

Protocols belonging to this category improve the performance of routing and reduce the control overhead by effectively utilizing the geographical information available.

LAR - Location-aided routing [22]

\section{CONCLUSION}

In this paper, the major issues involved in the design of a routing protocol and the different classifications of routing protocols for Adhoc wireless networks were described. The classifications of the Adhoc routing protocols is given in Table 1 in the Annexure. Comparison of Unipath routing protocols and the Multipath routing protocols are given in Table 2 and Table 3 respectively in the Annexure. The major challenges that an Adhoc wireless routing protocol must address are the mobility of nodes, rapid changes in topology, limited bandwidth, hidden and exposed terminal problem, limited battery power, time-varying channel properties, and locationdependant contention. The different approaches upon which the protocols can be classified include the classification based on the type of topology maintenance approach, the routing topology used, the use of temporal information, and the type of specific resource utilization considered for making routing decisions.

\section{ANNEXURE}

TABLE I. ClassificAtion OF THE AdHoc Routing Protocols 


\begin{tabular}{|c|c|c|c|c|c|c|c|c|c|}
\hline & 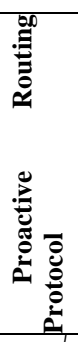 & 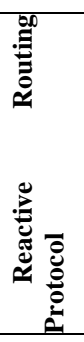 & 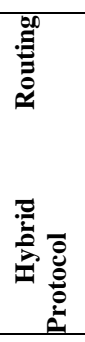 & 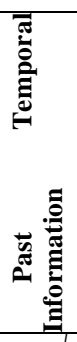 & 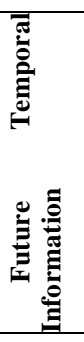 & 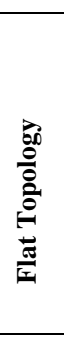 & 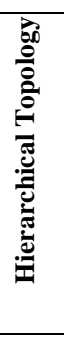 & 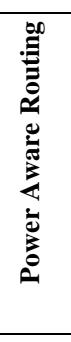 & 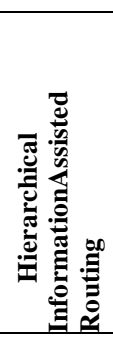 \\
\hline DSDV & $\sqrt{ }$ & & & $\sqrt{ }$ & & & & & \\
\hline WRP & $\sqrt{ }$ & & & $\sqrt{ }$ & & & & & \\
\hline CGSR & $\sqrt{ }$ & & & & & & $\sqrt{ }$ & & \\
\hline STAR & $\sqrt{ }$ & & & $\sqrt{ }$ & & & & & \\
\hline OLSR & $\sqrt{ }$ & & & & & & & & \\
\hline FSR & $\sqrt{ }$ & & & $\sqrt{ }$ & & & $\sqrt{ }$ & & \\
\hline HSR & $\sqrt{ }$ & & & $\sqrt{ }$ & & & $\sqrt{ }$ & & \\
\hline GSR & $\sqrt{ }$ & & & $\sqrt{ }$ & & & & & \\
\hline DSR & & $\sqrt{ }$ & & $\sqrt{ }$ & & $\sqrt{ }$ & & & \\
\hline AODV & & $\sqrt{ }$ & & $\sqrt{ }$ & & $\sqrt{ }$ & & & \\
\hline ABR & & $\sqrt{ }$ & & & & $\sqrt{ }$ & & & \\
\hline SSA & & $\sqrt{ }$ & & & & $\sqrt{ }$ & & & \\
\hline FORP & & $\sqrt{ }$ & & & $\sqrt{ }$ & $\sqrt{ }$ & & & \\
\hline PLBR & & $\sqrt{ }$ & & & & $\sqrt{ }$ & & & \\
\hline CEDAR & & & $\sqrt{ }$ & & & & & & \\
\hline ZRP & & & $\sqrt{ }$ & & & & & & \\
\hline ZHLS & & & $\sqrt{ }$ & & & & & & \\
\hline RABR & & & & & $\sqrt{ }$ & & & & \\
\hline LBR & & & & & $\sqrt{ }$ & & & & \\
\hline PAR & & & & & & & & $\sqrt{ }$ & \\
\hline LAR & & & & & & & & & $\sqrt{ }$ \\
\hline
\end{tabular}

TABLE II. COMPARISON OF THE UNIPATH ROUTING PROTOCOLS 


\begin{tabular}{|c|c|c|c|c|c|c|c|c|c|}
\hline & 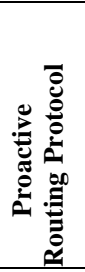 & 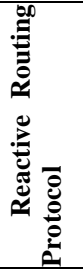 & 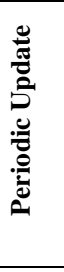 & 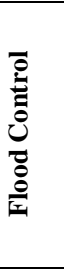 & • & 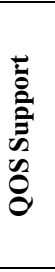 & 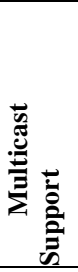 & 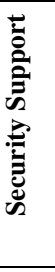 & 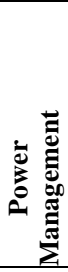 \\
\hline DSDV & Yes & No & Yes & No & Yes & No & No & No & No \\
\hline WRP & Yes & No & Yes & No & Yes & No & No & No & No \\
\hline GSR & Yes & No & Yes & No & Yes & No & No & No & No \\
\hline FSR & Yes & No & Yes & Yes & Yes & No & No & No & No \\
\hline AODV & No & Yes & No & Yes & Yes & No & Yes & No & No \\
\hline DSR & No & Yes & No & Yes & Yes & No & No & No & No \\
\hline CBRP & No & Yes & No & Yes & Yes & No & No & No & No \\
\hline
\end{tabular}

TABLE III. COMPARISON OF THE MUlTIPATH ROUTING PROTOCOLS

\begin{tabular}{|c|c|c|c|c|c|c|c|c|c|c|c|}
\hline & 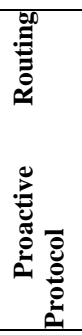 & 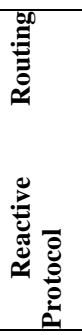 & 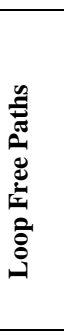 & 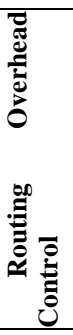 & 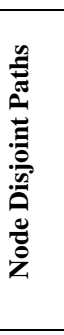 & 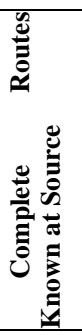 & 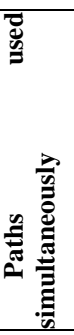 & 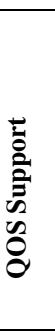 & 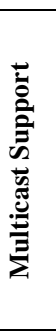 & 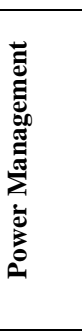 & 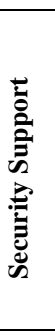 \\
\hline AOMDV & No & Yes & Yes & No & No & No & Yes & No & No & No & No \\
\hline AODVM & No & Yes & Yes & No & Yes & No & Yes & No & No & No & No \\
\hline SMR & No & Yes & Yes & No & No & Yes & Yes & No & No & No & No \\
\hline MSR & No & Yes & Yes & No & Yes & Yes & Yes & No & No & No & No \\
\hline
\end{tabular}

\section{REFERENCES}

[1] Keng Seng NG, Winston K.G.SEAH, "Routing security and Data Confidentiality for Mobile Adhoc Networks", Proceedings of the $5^{\text {th }}$ IEEE conference on Mobile and Wireless Communication Networks, pp $821-25$.

[2] C.Siva Ram Murthy and B.S.Manoj, "Adhoc Wireless Networks Architecture and Protocols", Prentice Hall 2004.

[3] C.E.Perkins and P.Bhagawat, "Highly Dynamic Destination-Sequenced Distance-Vector Routing(DSDV) for Mobile Compuetrs", Proceedings of ACMSIGCOMM 1994, pp 234-244, August 1994.

[4] S.Murthy and J.J.Garcia-Luna-Aceves, "An efficient Routing Protocol for Wireless Networks", ACM Mobile Networks and Applications Journal, Special Issue on Routing in Mobile Communication Networks, Vol.1, No.2, pp 183-197, October 1996.

[5] C.C.Chiang, H.K.Wu, W.Liu and M.Gerla, "Routing in Clustered MultiHop Mobile Wireless Networks with fading Channel", Proceedings of IEEE SICON 1997, pp 197-211,April 1997.

[6] J.J.Garcia-Aceves and M.Spohn, "Source-Tree Routing in Wireless Networks", Proceedings of IEEE ICNP 1999, pp 273-282, October 1999.
[7] T.H.Clausen,G.Hansen, Christensen and G.Behrmann, “ The Optimized Link State Routing Protocol, Evaluation through experiments and simulation", Proceedings of IEEE Symposium on Wireless Personal Mobile Communications 2001, September 2001.

[8] A.Iwata, C.C.Chiang, G.Pei, M.Gerla, and T.W.Chen, "Scalable Routing Strategies for AdHoc Wireless Networks",IEEE Journal on selected Areas in Communications, Vol 17, no 8, pp1369-1379, August 1999.

[9] T.W.Chen and M.Gerla, "Global State Routing : A New Routing Scheme for Adhoc Wireless Networks", Proceedings of IEEE ICC 1998, pp 171-175, June 1998.

[10] D.B Johnson and D.A Maltz, "Dynamic Source Routing in Adhoc Wireless Networks", Mobile Computing, Kluwer Academic Publishers, Vol 353, pp 153-181, 1996.

[11] C.E.Perkins and E.M.Royer, "Adhoc On-Demand Distance Vector Routing", Proceedings of IEEE Workshop on Mobile Computing Systems and Applications 1999, pp 90-100, February 1999.

[12] C.K.TOH,"Associativity-Based Routing for Adhoc Mobile Networks", Wireless Personal Communications, Vol.4, no.2, pp. 1-36, March 1997.

[13] R.Dube, C.D.Rais, K.Y.Wang, and S.K.Tripathi, "Signal Stability-Based Adaptive Routing for Adhoc Mobile Networks," IEEE Personal Communications Magazine, pp.36-45, February 1997. 
[14] W.Su and M.Gerla, "IPV6 Flow Handoff in Adhoc Wireless Networks Using Mobility Prediction", Proceedings of IEEE GLOBECOM 1999, pp. 271 -275, December 1999.

[15] R.S.Sisodia, B.S.Manoj and C.Siva Ram Murthy, "A Preferred LinkBased Routing Protocol for Adhoc Wireless Networks",Journal of Communications and Networks, Vol.4, no.1, pp.14-21, March 2002.

[16] P.Sinha, R.Sivakumar, and V.Bharghavan, "CEDAR: A Core Extraction Distributed Adhoc Routing Algorithm", IEEE Journal on selected areas in Communications, Vol.17, no.8, pp. 1454-1466, August 1999.

[17] Z.J.Haas, "The Routing Algorithm for the Reconfigurable Wireless Networks", Proceedings of ICUPC 1997, Vol.2, pp. 562-566 October 1997.

[18] M.Joa-Ng and I.T.Lu, "A Peer-to-Peer Zone-Based Two-Level Link State Routing for Mobile Adhoc Networks", IEEE Journal on Selected Areas in Communications, Vol.17, no.8, pp.1415-1425 August 1999.

[19] S.Agarwal, A.Ahuja, J.P.Singh, and R.Shorey, "Route-Lifetime Assesment-Based Routing(RABR) Protocol for Mobile Adhoc Networks," Proceedings of IEEE ICC 2000, Vol.3, pp.1697-1701 June 2000

[20] B.S.Manoj, R.Anandapadmanabha, and C.Siva Ram Murthy, "Link LifeBased Routing Protocol for Adhoc Wireless Networks", Proceedings of IEEE ICCCN 2001, PP.573-576, October 2001.

[21] S.Singh, M.Woo and C.S.Raghavendra, "Power-Aware Routing in Mobile Adhoc Networks", Proceedings of ACM MOBICOM 1998, pp.181-190 October 1998.

[22] Y.Ko and N.H.Vaidya, "Location-Aided Routing(LAR) in Mobile Adhoc Networks", Proceedings of ACM MOBICOM 1998, pp.66-75 October 1998.

\section{AUTHORS PROFILE}

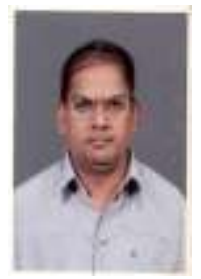

Udayachandran Ramasamy, born on 26.05.1956, completed his B.E (Electronics and Communication Engineering) in 1979, and M.E (Applied Electronics) in 1981 from P.S.G College of Technology, Coimbatore under University of Madras. He is currently Doctoral candidate at Vinayaka Missions University, Salem, India.

He has worked and carried out research in various institutions like REC, Tirchy, BIT, Bangalore, IOC, Assam, CIT, Coimbatore, VLBJACET, Coimbatore, Technical University of Novascotia, Canada Halifax, Concordia University, Montreal, Canada Institute Megatech, Kuala Lumput, Multimedia University, Kuala Lumpur.

He has 29 years of teaching and Research experience. His area of interest includes Mobile Adhoc Networks Distributed Computing a Grid Computing. At present he is working as Professor and Head, Department of Computer Science and Engineering, Sri Ramakrishna Institute of Technology, Coimbatore, Tamil Nadu, India.

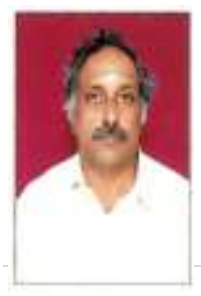

K.Sankaranarayanan, born on 15.06.1952, completed his B.E (Electronics and Communication Engineering) in 1975, and M.E (Applied Electronics) in 1978 from P.S.G College of Technology, Coimbatore under University of Madras. He did his Ph.D
(Biomedical Digital Signal Processing and Medical Expert System) in 1996 from P.S.G College of Technology, Coimbatore under Bharathiar University.

His areas of interest include Digital Signal Processing, Computer Networking, Network Security, Biomedical Electronics, Neural Networks and their applications, and Opto Electronic. He has more than 30 years of teaching experience and worked in various Government and Self financing Engineering Colleges.

At present he is working as PRINCIPAL at AKSHAYA College of Engineering and Technology, Coimbatore, Tamil Nadu, India. 